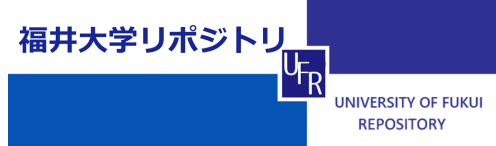

Real - t i me Opt i cal Moni tor i ng Syst em for Dye Col our and Concent $r$ at $i$ on

\begin{tabular}{|l|l|}
\hline 著者 & $\begin{array}{l}\text { UEDA Masahi } \mathrm{r} \text { o, M ZUNO Sanae, MATSUMRA Aki o, } \\
\text { SAKAN Fi j i o }\end{array}$ \\
\hline $\begin{array}{l}\text { j our nal or } \\
\text { publ i cat i on t i t l e }\end{array}$ & Opt i cs and Laser s i n Engi neer i ng \\
\hline vol une & 25 \\
\hline number & 1 \\
\hline page r ange & $13-23$ \\
\hline year & $1996-07$ \\
\hline URL & ht t p: //hdl . handl e. net /10098/1456 \\
\hline
\end{tabular}




\title{
Real-time Optical Monitoring System for Dye Colour and Concentration
}

\author{
Masahiro Ueda, Sanae Mizuno, Akio Matsumura \& Fijio Sakan
}

Faculty of Education, Fukui University, Bunkyo 3-9-1, Fukui 910, Japan

\begin{abstract}
A system for real-time monitoring of a dye has been constructed which enables us to measure the concentration and fluctuations of the three base colours. The method is based on light attenuation theory and its principle requires three different lasers having the three base colours. The system consisted of a semiconductor laser and argon ion laser for light sources, a photodiode for a light detector and a personal computer for data processing. Detection sensitivity for the dye concentration was a few $\mathrm{mg} / \mathrm{l}$ and the system can be applied for practical uses.
\end{abstract}

\section{INTRODUCTION}

In a dyeing process, it is the most important to dye a cloth with consistent colour. The tinting power of the dye, however, depends on its concentration, the material to be dyed, the temperature of the dye, and other parameters. The concentration of each colour dye in a dye mixture decreases with time. The colour dyes consumed must be replenished instantaneously to maintain the same tint. A measurement of a dye concentration is essential for this purpose. ${ }^{1}$

A typical method now used practically is spectrum analysis using a high power light source. This method, however, is expensive and takes tens of minutes to produce each measurement because a precise measurement requires a high spectral sensitivity and then dilution of the dye. A real-time measurement is thus practically impossible.

The principle of a real-time optical sensor for measuring dye concentration and the result of preliminary experiments using a 


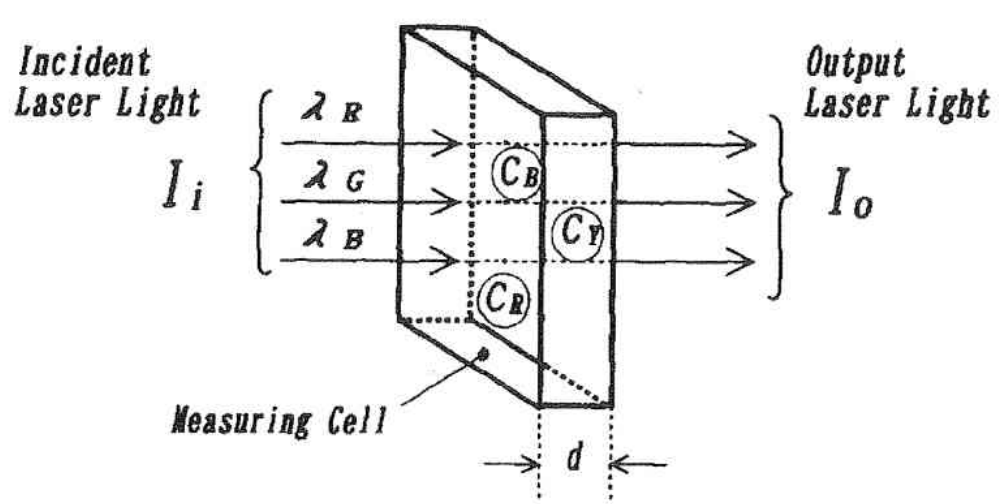

Fig. 1. Optical principle of the method. A part of the incident light of three primary colours is absorbed in a dye mixture consisting of the three primary dye colours.

semiconductor laser have been reported previously. ${ }^{2}$ In this paper, the flexibility of a sensor system is discussed from the viewpoint of practical use.

\section{PRINCIPLE AND METHOD}

Figure 1 shows the principle of the method. Laser light containing the three base colours is guided into a measuring cell. Part of the light is absorbed in the cell and the rest passes through the cell and is received on a photodiode. The intensity of the transmitted light can be expressed by Beer's law, ${ }^{3}$ if the dye is diluted, as

$$
\log \left(I_{\mathrm{o}} / I_{\mathrm{i}}\right)=-K n d \log (e), \quad n \equiv n_{x}+n_{\mathrm{o}}
$$

where $I_{\mathrm{i}}$ represents the power of the incident laser light, $I_{\mathrm{o}}$ the output power of the transmitted laser light, $K$ is an absorption coefficient, $d$ is the cell width. The overall concentration, $n$, includes the concentration of a dye, $n_{x}$, and a reduced concentration, $n_{\mathrm{o}}$, taking account of the optical glass of the cell walls and additives for dyeing, etc. As is shown in eqn (1), the absorbance defined by $\log \left(I_{\mathrm{o}} / I_{\mathrm{i}}\right)$ is in direct proportion to nd and the absorption coefficient can then be given as an absolute value of a gradient of the straight line absorption graph.

A dye can be mixed with three base colours, i.e. red, yellow and blue base dyes. Unknown parameters to be solved are thus the dye concentrations of each these three base colours. Three pieces of information are therefore essential for the solution. These can be obtained from the transmitted light power of three base colours. Light of wavelength $\lambda_{R}=670 \mathrm{~nm}$ from a semiconductor laser and wavelengths of $\lambda_{\mathrm{G}}=515 \mathrm{~nm}$ and $\lambda_{\mathrm{B}}=458 \mathrm{~nm}$ from a multiline Ar ion laser are used for the light sources. 
The absorption coefficient depends mainly on the wavelength of the light $\lambda\left(\lambda_{\mathrm{R}}, \lambda_{\mathrm{G}}, \lambda_{\mathrm{B}}\right)$ and the dye colour $C\left(C_{\mathrm{R}}, C_{\mathrm{Y}}, C_{\mathrm{B}}\right)$. The absorption coefficient has nine components:

$$
[\mathbf{K}(\lambda, \mathbf{C})] \equiv\left[\begin{array}{l}
K\left(\lambda_{\mathrm{R}}, C_{\mathrm{R}}\right), K\left(\lambda_{\mathrm{R}}, C_{\mathrm{Y}}\right), K\left(\lambda_{\mathrm{R}}, C_{\mathrm{B}}\right) \\
K\left(\lambda_{\mathrm{G}}, C_{\mathrm{R}}\right), K\left(\lambda_{\mathrm{G}}, C_{\mathrm{Y}}\right), K\left(\lambda_{\mathrm{G}}, C_{\mathrm{B}}\right) \\
K\left(\lambda_{\mathrm{B}}, C_{\mathrm{R}}\right), K\left(\lambda_{\mathrm{B}}, C_{\mathrm{Y}}\right), K\left(\lambda_{\mathrm{B}}, C_{\mathrm{B}}\right)
\end{array}\right]
$$

The dye toning mixed with three base colour dyes involves no chemical reactions. The light attenuation is then proportional to the product of each attenuation by the individual colour dyes. The superposition principle for light attenuation, therefore, can be applied to the present case where many colour dyes are mixed together. The assumption will be realized as discussed in Section 4.1. The transmitted light powers through the mixed dye are expressed as follows from eqns (1) and (2);

$$
\left[\log \left(\mathbf{I}_{\mathbf{o}} / \mathbf{I}_{\mathbf{i}}\right)\right]=-[\mathbf{K}][\mathbf{n}] d \log (e)
$$

where

$$
\left[\log \left(\mathbf{I}_{\mathrm{o}} / \mathbf{I}_{\mathrm{i}}\right)\right] \equiv\left[\begin{array}{c}
\log \left(I_{\mathrm{o}} / I_{\mathrm{i}}\right)_{\mathrm{R}} \\
\log \left(I_{\mathrm{o}} / I_{\mathrm{i}}\right)_{\mathrm{G}} \\
\log \left(I_{\mathrm{o}} / I_{\mathrm{i}}\right)_{\mathrm{B}}
\end{array}\right], \quad[\mathbf{n}] \equiv\left[\begin{array}{c}
n_{\mathrm{R}} \\
n_{\mathrm{G}} \\
n_{\mathrm{B}}
\end{array}\right]
$$

The concentration of each dye colour can be obtained as,

$$
[\mathbf{n}]=-[\mathbf{K}]^{-1}\left[\log \left(\mathbf{I}_{\mathbf{o}} / \mathbf{I}_{\mathbf{i}}\right)\right] /\{d \log (e)\}
$$

The concentration sensitivity of the sensor, i.e. the resolving power of the concentration, is defined by a small change of concentration due to a small change of the light power. It can, therefore, be given as

$$
[\Delta \mathbf{n}]=-[\mathbf{K}]^{-1}\left[\mathbf{\Delta I}_{\mathbf{0}} / \mathbf{I}_{\mathbf{i}}\right] / d
$$

where the negative signs in eqns (4) and (5) imply that an increase of light power corresponds to a decrease of the concentration.

Figure 2 shows the optical arrangement and the system of the sensor. Any wavelength of laser light can be selected by properly choosing the shielding plates $K_{2}, K_{3}, K_{4}$ and $K_{5}$. The output laser light through the measuring cell is focused on a photodiode and converted to an electric signal. The signal is amplified, sampled and digitized in 12 bits.

The maximum sampling frequency of the data acquisition is limited to about $1 \mathrm{kHz}$ by the $\mathrm{A}-\mathrm{D}$ converter. The digitized signal is used in eqn (4) to calculate the concentration and the results are displayed on the monitor. All the data used in this calculation are obtained by averaging by 80 samples. 


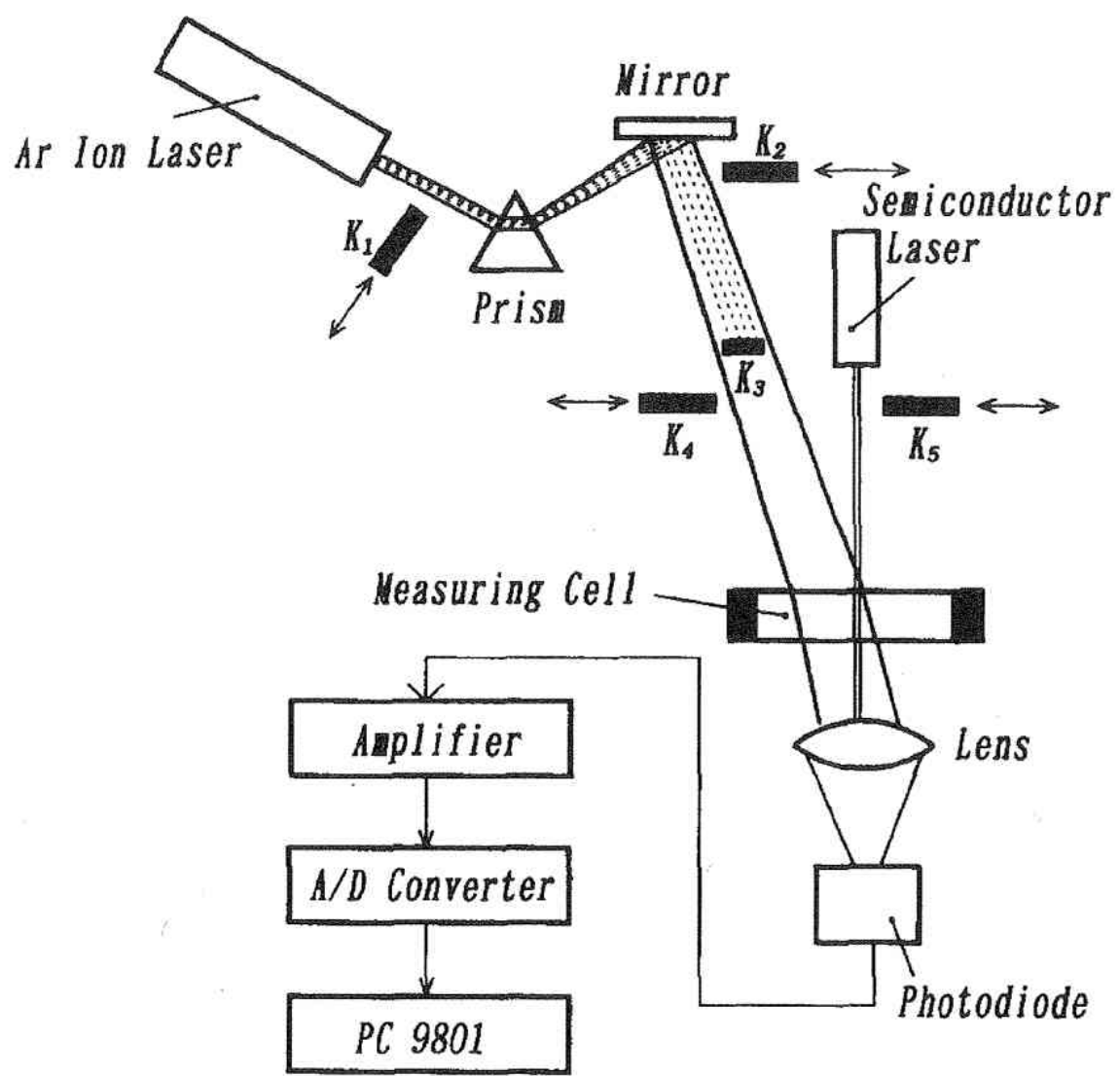

Fig. 2. Optical arrangement and sensor system.

The dyes used in the experiments are colour indication numbers of C.I. Reactive Red 112, C.I. Reactive Yellow 15 and C.I. Reactive Black 5. These are all ionized in water.

\section{EXPERIMENTAL RESULTS}

The output power of laser light was measured for a small change of the concentration. Figure 3 shows an example with a laser light of wavelength $\lambda_{\mathrm{R}}=670 \mathrm{~nm}$ and blue colour dyes. As seen in this figure, the logarithm of output laser power decreases almost linearly with an increase of dye concentration in a limited range of concentration. This indicates the validity of the Lambert-Beer's law expressed in eqn (1). The slope, $-K d$, has been calculated by the method of the least-squares fit. As seen in the figure, the absolute value of the slope, $K d$ decreases slightly as the concentration becomes high (the range of practical use). The small change of slope would be one of the major reasons for error of this method as discussed later in Section 4.2.

The value of the absorption coefficient in this case was calculated as $K\left(\lambda_{\mathrm{R}}, C_{\mathrm{B}}\right)=7.28 \mathrm{~g} /(1 \cdot \mathrm{cm})$ for $d=0.55 \mathrm{~cm}$. Similar experiments were 


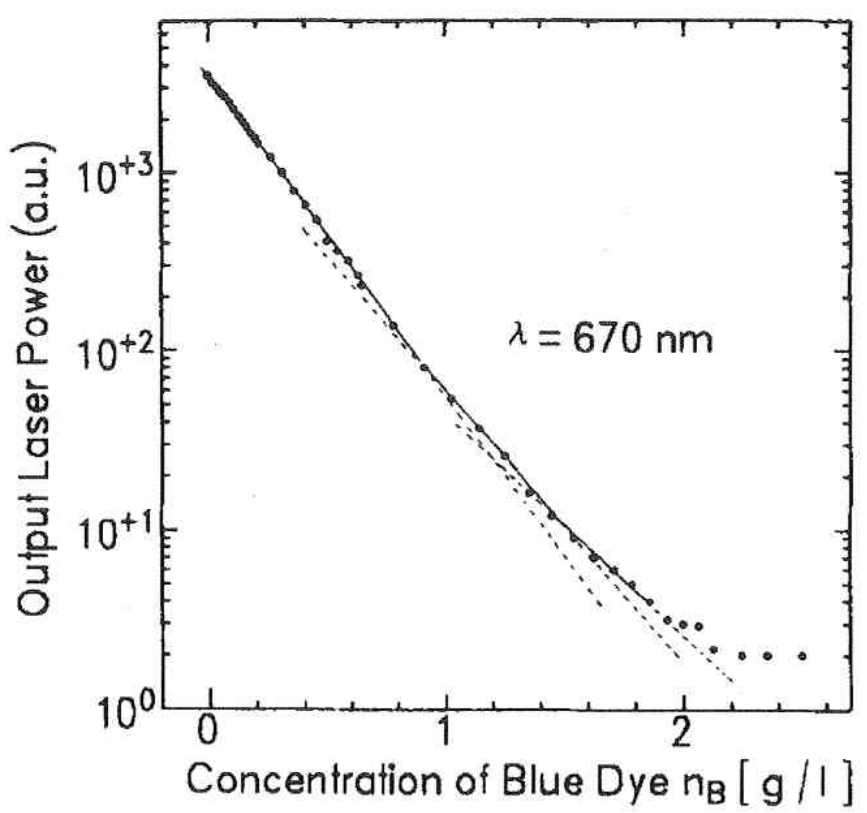

Fig. 3. Output power of the laser light with a wavelength $\lambda_{\mathrm{R}}=670 \mathrm{~nm}$ passing through a dye solution with various concentrations of blue dye.

run for all combinations of the three base colour lights and dyes. Table 1 shows all the data for $K$. The value changes on a large scale, depending on the combination of laser light and dye colours. This enables us to increase the sensitivity of the method to a level which is high enough for practical use.

Using the data in Table 1 in eqn (4), we obtain an expression for each concentration,

$$
\left[\begin{array}{l}
n_{\mathrm{R}} \\
n_{\mathrm{Y}} \\
n_{\mathrm{B}}
\end{array}\right]=\left[\begin{array}{ccc}
0.1820 & -0.0404 & 0.00399 \\
0.0305 & 0.0903 & -0.162 \\
-0.576 & 0.000148 & 0.0000720
\end{array}\right]\left[\begin{array}{l}
\log \left(I_{\mathrm{o}} / I_{\mathrm{i}}\right)_{\mathrm{R}} \\
\log \left(I_{\mathrm{o}} / I_{\mathrm{i}}\right)_{\mathrm{G}} \\
\log \left(I_{\mathrm{o}} / I_{\mathrm{i}}\right)_{\mathrm{B}}
\end{array}\right]
$$

An experiment of increasing each colour dye successively was carried out to confirm the reliability of the method. The dye concentration in the measuring cell filled with water was successively increased by droplets of high concentration dye. The output of the laser light was measured for each droplet and was used for the calculation of the dye concentration. Figure 4 shows an example of the calculated concentration for blue dye. As shown in this figure, the calculation concentration of blue colour dye changes only by the droplets of blue colour dye. The values of the change by both the droplets are about 0.009 and $0.016 \mathrm{~g} / \mathrm{l}$, respectively, which are in good agreement with the real change of $0.013 \mathrm{~g} / \mathrm{l}$. 
TABLE 1

Absorption Coefficients for all Combinations of Three Basic Lights and Dyes Absorption Coefficient

\begin{tabular}{|c|c|}
\hline$K\left(\lambda_{1}, C_{s}\right)$ & Heasured value $g /\left(l^{*} \mathrm{c}\right.$ (日) $)$ \\
\hline$K\left(\lambda_{R}, C_{R}\right)$ & 0.0360 \\
\hline$K\left(\lambda_{n}, C_{x}\right)$ & 0.00412 \\
\hline$K\left(\lambda_{R}, C_{B}\right)$ & 7. 28 \\
\hline$K\left(\lambda_{G}, C_{R}\right)$ & 109 \\
\hline$K\left(\lambda_{G}, C_{r}\right)$ & 2. 72 \\
\hline$K\left(\lambda_{a}, C_{B}\right)$ & 34.8 \\
\hline$K\left(\lambda_{B}, C_{R}\right)$ & 61.3 \\
\hline$K\left(\lambda_{s}, C_{\gamma}\right)$ & 27.4 \\
\hline$K\left(\lambda_{B}, C_{B}\right)$ & 20.8 \\
\hline
\end{tabular}

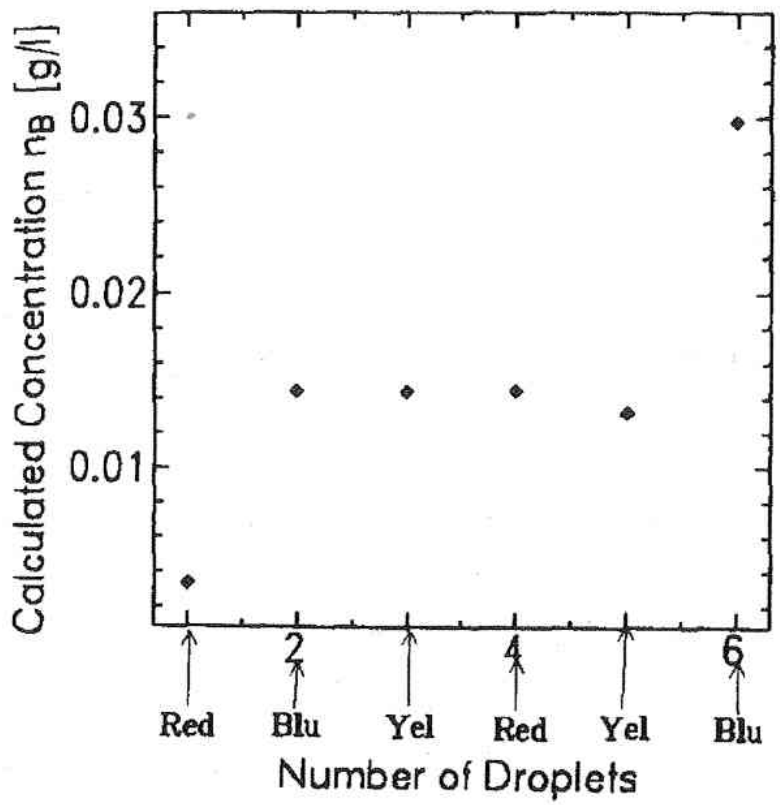

Fig. 4. Calculated concentration of blue dye for droplets of each colour of dye. 
The sensitivity of the concentration, shown in eqn (5), can also be obtained by using the data in Table 1 , as follows,

$$
\left[\begin{array}{c}
\Delta n_{\mathrm{R}} \\
\Delta n_{\mathrm{Y}} \\
\Delta n_{\mathrm{B}}
\end{array}\right]=\left[\begin{array}{ccc}
0.0789 & -0.0175 & 0.00173 \\
0.0133 & 0.0392 & -0.0703 \\
-0.250 & 0.0000640 & 0.000031
\end{array}\right]\left[\begin{array}{c}
\left(\Delta I_{\mathrm{o}} / I_{\mathrm{o}}\right)_{\mathrm{R}} \\
\left(\Delta I_{\mathrm{o}} / I_{\mathrm{o}}\right)_{\mathrm{G}} \\
\left(\Delta I_{\mathrm{o}} / I_{\mathrm{o}}\right)_{\mathrm{B}}
\end{array}\right]
$$

The sensitivity depends directly on the resolution of the data acquisition system for the output light power, $\Delta I_{\mathrm{o}} / I_{\mathrm{o}}$, which is given by the A-D converter if the sensitivity of the photodiode is sufficient. The tonal range of light output is between 1 and 256, that is, the resolving power is $1 / 256$, when the A-D converter with 8 bits is used. As an example, if the light power changes for three base colour of light are $\left(\Delta I_{\mathrm{o}} / I_{\mathrm{o}}\right)_{\mathrm{R}}=$ $0.01,\left(\Delta I_{\mathrm{o}} / I_{\mathrm{o}}\right)_{\mathrm{G}}=0.1$ and $\left(\Delta I_{\mathrm{o}} / I_{\mathrm{o}}\right)_{\mathrm{B}}=0.1$, the concentration sensitivities are calculated as $\Delta n_{\mathrm{R}}=-0.788 \mathrm{mg} / \mathrm{l}, \Delta n_{\mathrm{Y}}=-2.90 \mathrm{mg} / 1$ and $\Delta n_{\mathrm{B}}=$ $-2.49 \mathrm{mg} / \mathrm{l}$. These are in good agreement to the experimental result in Fig. 4.

\section{DISCUSSION}

\subsection{Validity of the superposition principle for light attenuation}

The reliability of the method is based on the superposition principle for the light attenuation. This can be considered to be valid from the fact that the dye mixing involves no chemical reaction. This has been examined experimentally. Figure 5 shows an example of light attenuation through the mixed dyes. The absorption coefficient $K\left(\lambda_{\mathrm{R}}, C_{\mathrm{R}}\right)$ is almost independent of the blue dye. This validates the adaptation of the superposition principle. A small change of $K\left(R_{\mathrm{R}}, C_{\mathrm{R}}\right)$ will yield an error as discussed in the next section.

\subsection{Error due to a small change of absorption coefficient}

In Fig. 4, a difference between true and calculated values of concentration, and also the concentration change of blue dye due to the gradual addition of red dye and yellow dye, are sources of error. The former was found to be about $3 \mathrm{mg} / 1$ and the latter about $1 \mathrm{mg} / \mathrm{l}$. Three major sources of error may be considered. The first results from light fluctuation due to ambient light. However, the ratio of the intensity of ambient light to the laser light has been made less than $1 / 1000$ by shielding the light receiving system. The value is less than the 


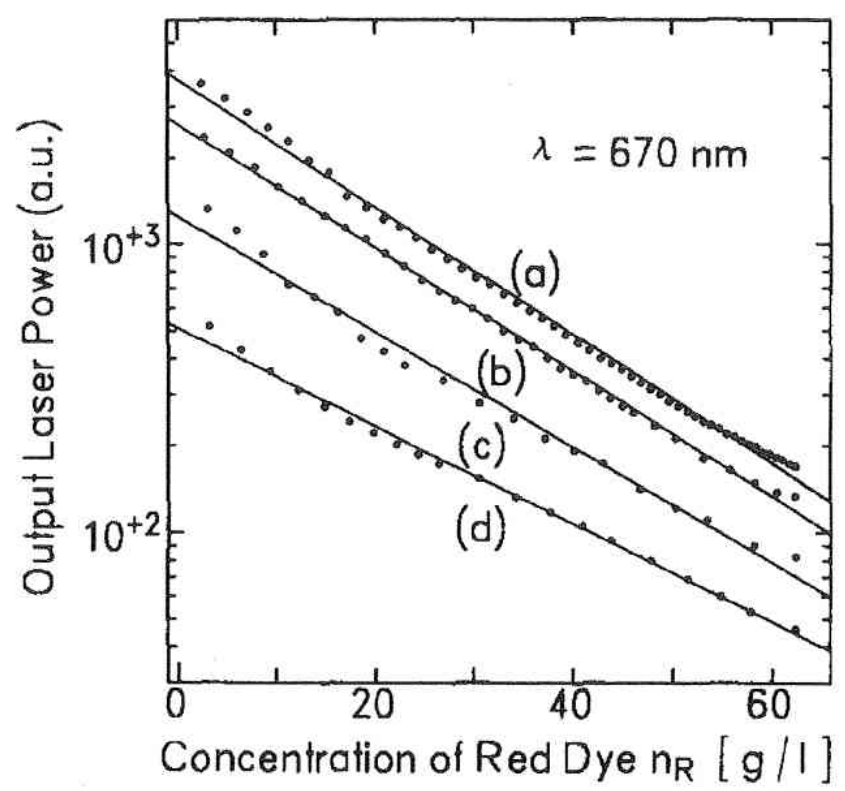

Fig. 5. Output power of the laser light with a wavelength $\lambda_{R}=670 \mathrm{~nm}$ passing through a dye solution with various concentrations of red colour dye added with and without blue colour dye: (a) without blue colour dye; (b) with blue colour dye of $n_{\mathrm{B}}=$ $0.0625 \mathrm{~g} / \mathrm{l} ;$ (c) $n_{\mathrm{B}}=0.125 \mathrm{~g} / \mathrm{l}$ and (d) $n_{\mathrm{B}}=0.345 \mathrm{~g} / \mathrm{l}$.

sensitivity and can be neglected. The second factor results from nonlinearlity between $\log \left(I_{\mathrm{o}} / I_{\mathrm{i}}\right)$ and $n$ in eqns (1) or (3), as shown in Fig. 3, when the concentration becomes high. We can, however, make the apparent concentration low enough by using a thin cell width as discussed in Section 3. The last results from a small change of the absorption coefficient due to the mixing of dye as described in Section 4.1. This will be a substantial problem for this method. A small change of concentration $[\Delta \mathbf{n}]$ due to a small change of absorption coefficient $[\Delta \mathbf{k}]$ can be calculated from eqn (3),

$$
[\Delta \mathbf{n}]=[\mathbf{k}]^{-1}[\Delta \mathbf{k}][\mathbf{k}]^{-1}\left[\ln \left(\mathbf{I}_{\mathbf{o}} / \mathbf{I}_{\mathbf{i}}\right)\right] / d
$$

where $[\mathbf{k}]^{-1}$ shows an inverse of the matrix $[\mathbf{k}]$. Thus, the change of dye concentration may be the same order of the change of the absorption coefficient.

\subsection{Adaptability for practical use}

Figure 6 shows an example of the output laser power for an extremely high absorption coefficient. The measurable range of the concentration is between 0 and $0.63 \mathrm{~g} / 1$ in this case. However, we can measure higher concentrations by using a thinner cell width $d$, because the absorption coefficient $K$ is the slope of a straight line between $\ln \left(I_{\mathrm{o}} / I_{\mathrm{i}}\right)$ and $n d$, as seen in eqn (1). That is, we can, apparently, make the concentration 


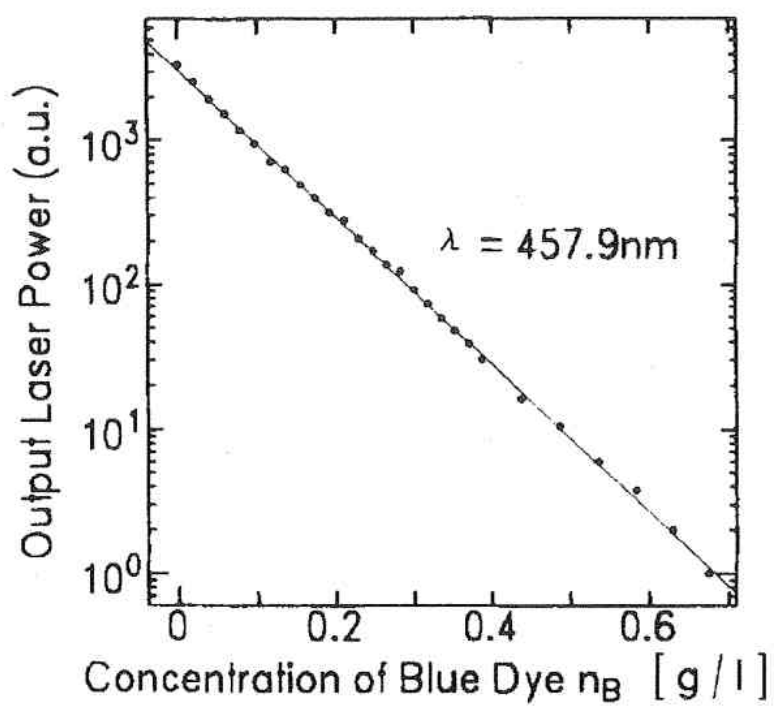

Fig. 6. Output power of the laser light with a wavelength $\lambda_{R}=458 \mathrm{~nm}$ passing through a dye solution with various concentrations of blue colour dye.

low by using a thin cell width. As an example, if a concentration range between 0 and $3 \mathrm{~g} / \mathrm{l}$ is required, it can, theoretically, be achieved only by making the cell width about $d=1 \mathrm{~mm}$. This will, however, cause an error due to a concentration fluctuation in such a thin cell width. One useful method to solve this problem may be to incorporate the light emitter and receiver in one unit as a sensor head using an optical fiber, as shown in Fig. 7. Practically only the sensor head is immersed in the dye solution.

\subsection{Optimum wavelength of the laser light}

Three base colours of lights with the wavelengths $\lambda_{\mathrm{R}}=670 \mathrm{~nm}, \lambda_{\mathrm{G}}=$ $515 \mathrm{~nm}$ and $\lambda_{\mathrm{B}}=458 \mathrm{~nm}$ were used for the light sources in this study. However, another wavelength may also be used effectively. A large difference in absorbance, defined by $\log \left(I_{\mathrm{o}} / I_{\mathrm{i}}\right)$, for each wavelength and dye colour is desirable for high sensitivity. Figure 8 shows the absorption spectrum for three base colour dyes. It may be concluded from Fig. 8 that the desirable wavelengths of the laser lights are $430 \mathrm{~nm}$, $460 \mathrm{~nm}, 510 \mathrm{~nm}, 540 \mathrm{~nm}$ and $580 \mathrm{~nm}$. Any three wavelengths in these five wavelengths, e.g. $460 \mathrm{~nm}, 510 \mathrm{~nm}$ and $580 \mathrm{~nm}$, will be sufficient for determining three unknown parameters as discussed in this paper. 


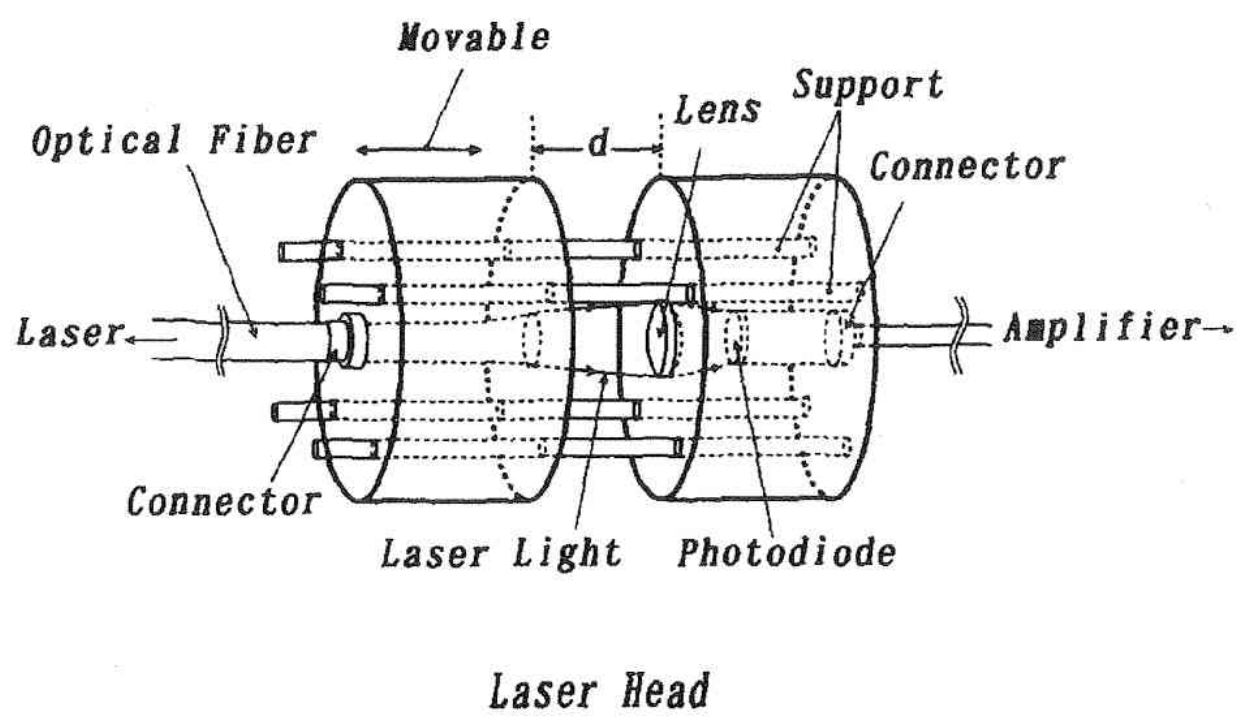

Fig. 7. Illustration of a proposed optical sensor head.

\section{CONCLUSION}

An optical monitoring system has been developed for dye colour and concentration detection. The system consisted of a semiconductor laser and an Ar ion laser, a photodiode as a light detector and a personal computer.

The system can be used effectively for monitoring or detecting a small change of dye concentration. The concentration sensitivity of the

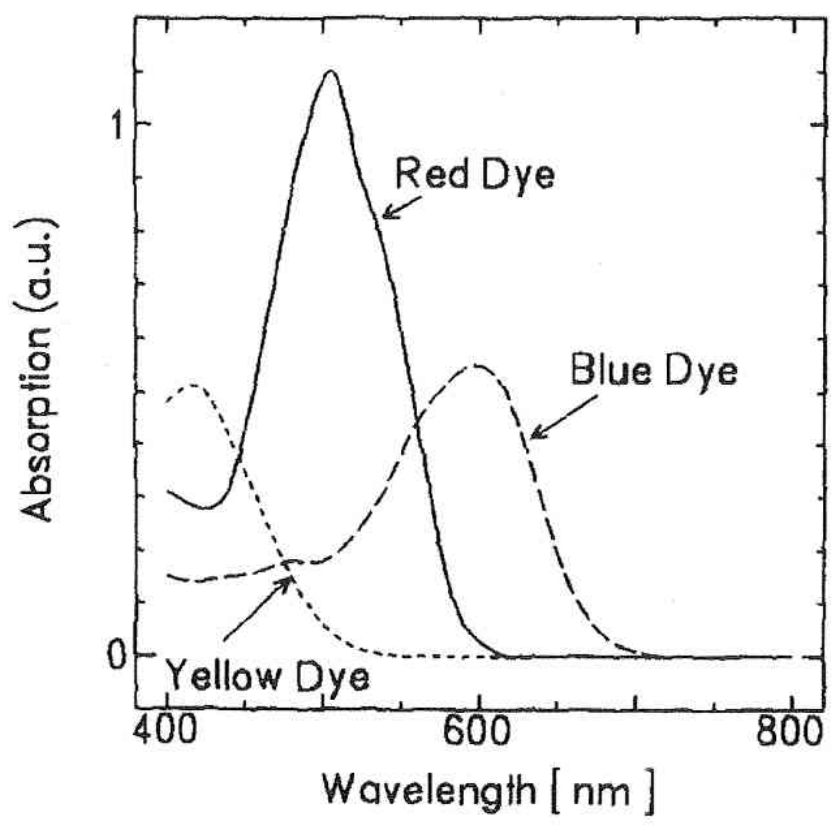

Fig. 8. Absorption spectra of three base colour dyes. 
method was about a few $\mathrm{mg} / \mathrm{l}$ and may be satisfactory for dyeing machines presently on the market.

The method is based on the principle of light attenuation and can only be applied for the ionic dyes studied in this paper. Another method based on light dispersion is now under study for dispersing dyes.

\section{REFERENCES}

1. Tsuda, H., A streamlining production by realtime control of dye adsorption. Indust. Technol., 297 (1994) 472-4 (in Japanese).

2. Ueda, M., Mizuno, S. \& Matsumura, A., Realtime optical sensor for dye color and concentration detection. Laser Eng., 22 (1994) 828-33 (in Japanese).

3. Kubo, R. \& Takahashi, H. (eds), Encyclopedia for physics and Chemistry, 3rd edn. Iwanami, 1976, 1400 pp. (in Japanese). 